\title{
Das älteste Ordinationsformular der lutherischen Kirche.
}

\author{
Von Paul Vetter.
}

Im jüngst veröffentlichten 38 . Bande ${ }^{1}$ ) der Weimarer Ausgabe von Luthers Werken wird ron P Drews ein Schriftstuck als das älteste Ordinationsformular unserer protestantischen Kirche anfs neue bezeichnet and heransgegeben, das jedem Kenner des Spracbgebranchs des Reformators zam mindesten als höchst verdächtig erscheinen maß.

Gemäß seiner Auffassung von der Tanfe und dem allgemeinen Priestertum batte Luther ursprïnglich an eine feierliche Ordination als Ersatz für die katholische Priesterweihe nicht gedacht. Das Haupterfordernis für den Geistlichen seiner Kirche sah er in der Vokation durch die weltliche Obrigkeit, die Vertreterin der Gemeinde. So einfach und folgerichtig eine solche Anschauung auch sein mochte, für die Praxis ergaben sich aus ihr mancherlei Schwierigkeiten. Der Wurde des geistlichen Amtes trug sie zu wenig Kechnung, und bald sah sich der Reformator zu Zogeständnissen an diese genötigt. Die Konzessionen berücksichtigen zunächst Einzelfälle. Ende der zwanziger Jahre muß sich aber in den führenden Wittenberger Theologenkreisen die Überzeugung durchgerangen haben, dab es im Interesse der Stellung der Geistlichen ibren Gemeinden gegenuber liege, wenn sie darch einen Ordinationsakt in den Besitz ibres Amtes gelangten. Das bezengen die Kirchenordnungen Bugenhagens in Hamburg und Litbeck.

In Wittenberg selbst hielt man zanächt an dem alten Branche noch fest, bis 1535 der Kurfurst die Einführang einer Ordinationshandlang anordnete ${ }^{2}$ ). Wohl weniger die

1) S. $401 \mathrm{ff}$. Hier auch die einschlägige Literatur.

2) Mentz, Johann Friedrich der Großmïtige III, 240. 
Gewissensnot der Geistlichen als die Haltung der Gemeinden gegenuber den ungeweihten Pfarrern muBten das landesherrliche Kirchenregiment darauf hindrängen, für einen Ersatz der Priesterweihe za sorgen. welche die Bischöfe der alten Kirche den Anhängern Luthers naturgemä $B$ versagten. so erschien am 12. Mai 1535 die denkwürdige Anordnung Johann Friedrichs, die fortan eine Ordination fur die neu einzusetzenden Geistlichen verlangte, welche die Priesterweibe nicht erbalten hatten. Inwieweit Latber selbst an diesem karfurstlichen Befehle beteiligt ist, läßt sich leider nicht sagen. Das sonst so reichlich fließende Aktenmaterial des Ernestinischen Gesamtarchivs in Weimar rersagt hier röllig. Als sicher kann angenommen werden, daB er seine Zastimmang nicht rerweigert hat. Verlangte seine Anffassung einen solchen Akt anch nicht unbedingt, die Erfahrang batte inzwischen gelehrt, da $B$ er nötig war. Bemerkenswert ist, and das wird wohl weniger auf Lather als anf die Kreise, die binter ihm standen und ihn beeinflaBten, zuruckgehen, daB nicht den Superintendenten, sondern der theologischen Fakultät zn Wittenberg das Recht der Ordination ubertragen wurde. lhre Bedeutang fur die neue Kirche erhielt dadarch eine wichtige Förderang, zamal noch in demselben Jabre 1535 anch die Prufung der za Ordinierenden, die ursprtinglich den Superintendenten uberlassen worden war, von diesen auf sie uberging. Mit Recht hat Drews daranf hingewiesen, daß schon vor dem 20. Oktober 1535 in Wittenberg Ordinationen erfolgt sind. Johann Friedrich hätte auch eine Verletzang seines Befehls nicht angerigt hingehen lassen. Zam Ordinator bestellte die Fakultät noch im Laufe des Jahres Bugenhagen. Doch hat Lather, der zunächst in Frage gekommen war, anch später noch, meist in Vertretung fur ihn, ordiniert. Die Ordination erfolgte gewöhnlich Sonntags im Anschlub an die Predigt, in der zar Furbitte fur die zu Ordinierenden aufgefordert warde.

In welchen Formen rollzog sich nan die Ordination? Da liegt die eigenartige Tatsache vor, daB bis zam Jahre 1539, abgesehen von einem lateinischen Formular, das für die des Deatschen Unkundigen bestimmt war und ganz sicher nicht in die erste Zeit der nenen Einrichtong fällt, nicht 
weviger als funf Ordinationsformulare vorhauden sind. Dazu kommt noch ein Bericht uber eine Ordination Luthers aus dem Jahre 1537. Es ist nicht recht verständlich, wie Drews die Erıählung der Tischreden ${ }^{1}$ ), die ihn enthalten, ein Formular nennen kann. Wenn es auch möglich ist, aus ihr ein Stuck der Ordinationshandlung zu rekonstruieren, so darf das Gewonnene angesichts der ganzen Art und Weise, wie die uns vorliegenden Tischreden entstanden sind, nur mit größter Vorsicht verwertet werden.

Keines der Formalare, die zam Teil nicht unbeträchtlich voneinander abweichen, nennt Luther als seinen Verfasser; sie gebeu meisi an, das Ordinationsformular der Wittenberger Kirche za sein. Unter solchen Umständen wirft sich ron selber die Frage auf: Sind sie als Arbeiten Luthers zu betrachten? Es darf von vornherein angenommen werden, daB bei der Wichtigkeit der neuen Einrichtang der Reformator selbst die Formen für sie schuf. Dazu komnt für das Formular $\mathrm{R}$ ein Zeugnis Rörers, das freilich wohl aus späterer Zeit stammen durfte. Ob sogleich bei der Einfubrung 1535 eine Formel aufgesetzt worden ist, entzieht sich unserer Kenutnis. Unmöglich wäre es nicht, wenu Luther sich zunächst an ein gewisses Schema gehalten hätte, und wenn erst später, als man von auswärts nach dem Ordinationsformular begehrte, die Niederschrift erfolgt wäre. Will man nun die Formeln $\mathrm{C}, \mathrm{F}, \mathrm{H}$ und I sämtlich auf Luther zuriuckfuhren, so kunstatiert man damit die Tatsache, dal er is den Jahren 1535-1538 mindestens zweimal sein Formular wesentlich verändert hat. Die für die Wittenberger Kirche seit 1539 obligatorische Formel $R$ hat Bugenhagen nach seiner Ruckkehr aus Dänemark durch Streichungen sich zurechtgemacht.

Die zeitliche Aufeinanderfolge dieser Formulare steht nar zum Teil fest. Wäbrend $K$ ins Jahr 1538 oder 1539 gehört ${ }^{2}$ ), bekennen $\mathrm{C}$ und $\mathrm{F}$ sich als Formeln aus dem

1) Förstemann, Luthers Tischreden II, $383 \mathrm{f}$. vgl. dazu Enders, Luthers Briefwechsel XI, $227 \mathrm{f}$.

2) Rietschel, Luther und die Ordination. 2. Aufl. 1889. S. 10. Das Formular $\mathrm{R}$ ist weiter nichts als eine, und zwar recht konservative Bearbeitung von $F$, das danit für den Ordinator etwas bequemer gemacht wird. Die eigentliche Ordinationsrede ist, allerdings in wenig 
Jahre 1538. $\mathrm{H}$ and $\mathrm{I}$ dagegen sind undatiert. $\mathrm{C}$ ist ron den Kulmbacher Predigern Schnabel and Eberhard aus Wittenberg in die fränkische Heimat mitgenommen worden. Sie versichern ausdrucklich, das Formular von Lather and Melanchthon erhalten zu haben. Merkwurdigerweise fehlt ihm der SchluB. der sich bei allen anderen Formeln findet. Entweder haben ihn die Reformatoren fur weniger bedeutsam und eutbehrlich angesehen, oder, und das ist das Wahrscheinlichere, es liegt das Versehen eines Schreibers vor. Da die Anwesenheit der Kulmbacher in Wittenberg im Sommer 1538 stattfand, so wird ihnen etwa um die Mitte

geschmackroller Weise, gekürzt, dafür ist die Segnung der Ordinanden mit dem Kreuze, die in $F$ nicht ausdrücklich erwähnt wird, hinzngefügt worden. Der Ordinationshandlung wird sie wohl anch vor $R$ nicht gefehlt haben. Wann $R$ entstanden ist, läbt sich mit Bestimmtheit nicbt sagen, entweder noch $1 \overline{3} 38$ oder in der ersten Hälfte ron 1539. Bugenhagen fand bei seiner Rückkehr aus Dänemark das Schriftstück wohl bereits vor. Wer der Redaktor gewesen ist, kann ebenfalls nicht mit Sicherheit ermittelt werden. Luthers Verfasserschaft ist wenig wahrscheinlich. Es ist nicht abzusehen, warum er, der von Mitte 1537 bis Mitte 1539 ordinierte, das Formular von 1538 in dieser Weise verändert haben sollte. Er für seine Person kann doch ein solches Bedürfnis schwerlich gehabt baben. Eher könnte man an Rörer denkeu, der 1538 mehrere Male für Luther ordinieren maßte, und der wohl den Wunsch gehabt haben kann, sich das Formular, seinen Bedürfnissen entsprechend, etwas zurechtzumschen. Kolde hat gemeint, daß Bugenhagen der Redaktor von $\mathrm{R}$ sei. Seine Gründe sind nicht durchschlagend. Was binderte Bugenhagen nach seiner Rückkehr nach Wittenberg, das Formular $F$ anznnehmen? Und wenn er wirklich der Verfasser von $\mathrm{R}$ war, waram dann die Ändernugen an ihm, als deren Urheber ihn Rietschel nachgewiesen hat? Der Hauptgrund holdes, daB die Änderungen Bugenhagens an $R$ sich in derselben Linie bewegen wie die Veränderungen, die aus $F \mathrm{R}$ gemacht baben, ist wohl an sich richtig, aber nicht in der Weise beweiskraftig, wie Kolde annimmt. Denn Bugenhagen kann ebensogat an einer fremden Arbeit, die ihm nicht weit genug ging, diese Ändernngen vorgenommen haben als an der eignen. Jedenfalls ist eins klar. Mit den vorhandenen Mitteln läßt sich die Frage nach der Verfasserschaft von $R$ nicht beantworten. DaB Lutber der Redaktor gewesen sei, scheint mir wenig wahrscheinlich. Das Zeugnis Rörers will dabei nicht viel bedeuten; denn auch in seiner Fassung ist $R$ als geistiges Eigentum des Reformators anzusehen. Für Bugenhagens Autorschaft spricht gleichfalls nicht viel. Ein Dritter, dessen Namen noch za ermitteln ist, mag wohl der Redaktor gewesen sein. 
des Jahres das Formular eingehändigt worden sein. F findet sich in den Akten der Freiberger Visitation von 1538. Wie es in sie kam, ist nicht ohne weiteres ersichtlich. Falsch ist, was Drews hieruber sagt. Nicht Jonas hat es nach Freiberg gebracht, ist er 1538 doch gar nicht in Herzog Heinrichs Lande Visitator gewesen. Drews folgt hier Burkhardts and Seidemanns Irrtam. der indes längst beseitigt ist $^{1}$ ). Anch Spalatin hat es sicher nicht mitgebracht. $\mathrm{Za}$ welchem Zweck anch? In Wittenberg hat man 1538 gar nicht daran gedacht, dem Freiberger Saperintendenten oder den Visitatoren das Recht der Ordination für Herzog Heinricbs Land $\%$ gewähren. Schenck hat allerdings etwas derartiges in Plane gehabt and za diesem Zwecke, vielleicht durch den Wittenberger Mädchenschallehrer Eyselein ${ }^{2}$ ), sich das Schriftstuck verschafft. An einer Verwirklichang seiner Hoffnongen hat ihn dann sein Sturz verhindert. Spalatin wird bei der Visitation das Formular unter den Papieren der Freiberger Superintendentur gefunden and den Akten beigefugt haben. Denkbar ist es auch, dab es erst nach Schencks Abreise eintraf und auf diese Weise in die Hände des Visitators kam. Da Schenck Anfang Jali Freiberg verließ, ist dieser Monat der spätest mögliche Termin für die Entstehang von $F$. Es fällt höchstwahrscheinlich in die erste Hälfte des Jahres und damit zeitlich noch ror C.

Nicht so einfach wie bei $\mathrm{K}, \mathrm{C}$ und $\mathrm{F}$ ist die Datierung von $\mathrm{H}$ and I. Das letztere warde 1895 von Rietschel zam ersten Male aus einem Jenaer Kodex heransgegeben. Die Voraussetzangen, anf denen seine Datierang des Formulars beruht, sind von Drews als unrichtig nachgewiesen worden. Das Jahr 1537, das Kietschel als Entstehungszeit annahm, bedurfte daher weiterer Stützen, die bisher fehlen. Drews hat nicht so unrecht, wenn er unter solchen Umständen auf eine Datierung überhaupt verzichtet. Um so unverständlicher ist es mir, dab er es mir als schweren metbodischen Fehler

1) Vgl. Kawerau, Briefwechsel des Justus Jonas II, XXXIXf., Brandenburg im N. Archiv t. Sächs. Gesch. N. F. XVII, 155; Vetter ebenda XXXII, $38 \mathrm{ff}$.

2) Vgl. über ibn Kawerau in Theol. Studien und Kritiken 1913 S. $\mathbf{5 3 7 .}$ 
rorwirft, daB ich die Datierung Rietschels, der I vor $\mathrm{C}$ ansetzt, ignoriert habe. Er tibersieht, daß der Ort, wo ich F veröffentlichte, za einer solchen Auseinandersetzung nicht geeignet war; ich mußte mancherlei karz abtan, wofür ich an anderer Stelle den ausfuhrlichen Beweis gefuhrt bätte, und mich damit begnugen, die eigene Ansicht zu bringen. Weiter verstehe ich nicht, wie er nach seiner eignen Beweisfuhrung sagen kann, Rietschel habe den Nachweis gefuhrt, dab I älter sei als C. Worin besteht dieser Nachweis, den ubrigens schon sehling angezweifelt hat? Rietschel weist zunächst darauf hin, dab der Anfang von I nachlässiger abgefabt sei als der von $C$ und $R$, wie er auch sonst die Redaktion von $I$ als nachlässig bezeichnet. Das ist gewiß ein wertvolles Argument fur Lather als Verfasser, der ja bekanntlich bei der Abfassung so wichtiger Aktenstucke ein nachlässiger Arbeiter za sein pflegte! Weiter macht er darauf aufmerksam, dab das Einleitungsgebet in I ausfuhrlicier skizziert ist als in den beiden genannten Formularen, und daß eine zwiefache Intonation zur Answahl gegeben ist. Der Schlaß freilich, den er hieraus ziehen will, ist sehr gewagt; dann muB man auch aus der Tatsache, daß das in den Tischreden enthaltene Formelfragment $S$ eine reichere Auswahl der zu verlesenden Texte bietet als alle ubrigen Formulare, den Schla $B$ gestatten, daB wir in ihm ein Stuck des ältesten Formulars vor uns bätten. Man bedenke doch, daB Luther das Original des Ordinationsformulars abgefabt hat and daB es bei seiner schriftstellerischen Eigenart sich kaum durch besondere Breite ansgezeichnet haben wird. Aus der Ausfuhriichkeit von I möcbte man daber wohl eher folgern, dab sie bestimmt war, in das knapp gefaßte lutherische Original etwas Bewegungsfreiheit für den Ordinator zu bringen.

Weiter unterscheidet sich $I$ vor $C$ und $R$ dadarch, $d a B$ es vor den zu verlesenden Texten eine lateinische Ansprache des Ordinators bringt, die in des beiden anderen Formularen fehlt. Rietschel meint, sie trage ein durchaus gesund lutherisches Gepräge. Das ist richtig, soweit es den Gedankeninhalt angeht. Sehen wir uns dagegen die Form an, die Diktion, so werden wohl wenige Kenuer von Lathers Sprache diese Rede als Eigentum des Reformators anerkennen wollen. 
Dazu kommt, daB sie inhaltlich auch nicht das geringste gemein hat mit der in $\mathrm{C}$ und $\mathrm{K}$. Weiter ist es iberaus merkwürdig, dab die Ordinationsrede vor den Texten gebracht wird, auf denen sie beruhen soll. Auf diese Texte folgt nan noch das Fragment einer zweiten Ordinationsrede, die beweist, daB dem Verfasser von I die Ordinationsrede Luthers in $F$ and $C$ bekannt gewesen sein maß. Die Form dieses Fragmentes ist so lässig, daB die Verfasserschaft des Reformators unter allen Umständen abgelehnt werden maB Endlich was soll die lateinische Form? Waram gibt der Verfasser von 1 Luthers Ansprache nicht im Original wieder? Hängt damit etwa zusammen, da $B$ die Verpflichtungsfrage an die Ordinanden und deren Gelöbnis feblen? Das eine durfte jedenfalls aus dem Gesagten hervorgehen. Dieser Teil von 1 kann so, wie er ans vorliegt, nicht Lathers Arbeit sein. Damit fallen alle weiteren Vermatungen Rietschels ron selbst in sich zasammen. Der Schluß von I stimmt wieder mit $\mathrm{C}$, soweit es reicht, and weiter mit $\mathrm{F}$ uberein.

Aus den vorstehenden Erörterungen dürtte hervorgehen. dab wir es in I nicht mit einem offiziellen Formulare der Wittenberger Kirche zu tan haben können. Welcbem Zwecke es gedient bat, ist uicht ersichtlich. Auch die Vermatung, dab es der Entwurl Luthers zu einer solchen Formel sei, muß angesichts des Zustandes der ersten Hälfte abgelehnt werden.

Neben I liegt nun noch eine funfte Formel ror, die Drews heransgegeben hat und die er $\mathrm{H}$ nennt. Er sieht in dem Schriftstitcke, das er Buchwald verdankt, die Nachschrift des Schlusses einer Predigt Luthers mit daranffolgendem Ordinationsformular. Im ersten Teile ${ }^{1}$ ) baben wir es allerdings mit der lateinischen Version eines Predigtfragments zo tun, das Lather zugesprochen werden darf. Der Nachschreiber hat freilich von der Eigenart der lutherischen Diktion gar viel verwischt, aber es ist doch genug vorhanden, um deu Schluß zu rechtfertigen, daß wir die Einleitang zu einer Ordination, die sich an eine Predigt Luthers ankntipfte, vor uns haben. Drews hat auch die Frage aufgeworfen, ob

1) D. Martin Luthers Werke. Weim. Ausg. 41, $762 \mathrm{f}$. 
etwa eine Tischrede vorliegt; er hat sie mit Recht verneint. Mit den Worten Commendatur igitur beginnt darauf der zweite Teil des Schriftstuckes, den sein Herausgeber ein Ordinationsformular Luthers nennt. DaB er in keinem Zasammenhange mit dem Vorausgegangenen stehen kann, zeigt seine Form. Im ersten Teile haben wir die Einleitung eines Bericbts itber eine bestimmte Ordinationshandlung Lathers ror ans. Wie der Ordinator gesprochen hat, wird ans in direkter Rede, wenn auch in lateinischer Ü̉bersetznng wiedergegeben. Mit dem Anfange des zweiten Teiles beginnt ein merkwurdiges Gemenge; halb erhalten wir eine allgemeine Schilderang einer Ordinationshandlang, halb das Formular einer solchen. Indikativ and Konjunktiv wechseln als Modi in banter Reihe miteinander ab. Die Namen der Ordinanden werden nicht genannt, ein deatliches Zeichen daftur, daß dieser Teil des Schriftstickes mit der Einleitung nichts gemein haben kann ${ }^{1}$ ). Wie deren Fortsetzung ansgesehen haben muß, das läßt sich onschwer erraten. Sie ist die Beschreibung einer bestimmten Ordinationshandlung gewesen. Es werden die Namen der Ordinanden angefuhrt worden sein, fur das Wort Ordinator wird uberall Dr. Martinns ge. standen baben usw. So wie der zweite T'eil aussieht, ist es jedenfalls anmöglich, ibn mit dem ersten za einem Ganzen zasammenzaschweißen. Wir baben in ibnen völlig verschiedenartige Bestandteile, die nur durch die Partikel igitar miteinander verbunden sind, and schlecht genug daza. Denn die Anfforderung zum Gebet für die Ordinanden und das gesamte Predigtamt, mit der $\mathrm{H}$ anfängt, ist ja schon in der Einleitung an die Gemeinde ergangen. Der ganze erste Teil von $H$ ist also völlig gegenstandslos, denn was er verlangt, das ist eben in ausfubrlichster Weise bereits gesehehen.

Wie die beiden Bestandteile des Scbriftstitckes zueinander gekommen sind, läßt sich nur vermuten. Der erste ist wohl zweifellos die Einleitung za einer Ordinatioushandlung Lathers, die von irgendeinem Zeitgenossen des Reformators

1) Drews will allerdings in der Tatsache, daB die Namen der Ordinanden nicht genannt werden, keinen Grund dafür sehen, daß die beiden Teile nicht zusammengehören können. Eine Begründung seiner Angicht hat er freilich nicht gegeben. 
niedergescbrieben warde. Aber er reicht nar bis zu den Worten in nostra infirmitate ostendat, sei es daB der Nachschreiber von hier ab an weiteren Aufzeichnungen verhindert war, sei es dab diese verloren gegangen sind. Später hat dann jemand das Bedurfnis emplunden, die Lücke auszafüllen, and sich dazu eines Ordinationsformulars bedient, das ihm zugänglich war.

Ist nun $\mathrm{H}$ ein Ordinationsformular Lathers für die Wittenberger Kirche?

Vergleichen wir $\mathrm{H}$ mit den ubrigen Formularen, so seben wir auf den ersten Blick, da $B$ es mit I aufs engste verwandt sein mab, während es deu anderen fernersteht. Zwei Möglichkeiten sind damit fur $\mathrm{H}$ und I gegeben: entweder ist das eine von dem anderen abbängig, oder beide gehen anf ein gemeinsames Original zurlick. Das letztere anzunehmen ist von vornherein sebr verlockend; denn beide Schriftstucke tragen die Sparen groBer Fluchtigkeit, und gar manche Abweichung läßt sich dadurch erklären. Drews freilich will von einer solchen Herkuntt von $\mathrm{H}$ nichts wissen. Ihm, dem es darauf ankommt, die Priorität seines Formulars vor allen anderen darzutan, ist $\mathrm{H}$ die älteste aller vorbandenen Formeln und wohl noch dem Jahre 1535 angehürig, dazu in jeder Weise genuinlatherisch.

Den Beweis dafur sucht er in folgender Weise zu fuhren. Zunächst nimmt er mit Rietschel an, daß I älter als alle ubrigen Formulare sei. Nan bleibt nur noch der Nachweis ubrig, da $B$ vor $I$ anzusetzen ist. Nicht weniger als 13 Gründe hat er dafur angefuhrt, nicht ein einziger davou ist irgendwie beweiskräftig. Seine Methode ist derart, dab man alles und nichts mit ihr beweisen kann. Jede $A b-$ weichung seines Formulars von den anderen spricht fur dessen Priorität, and wo gar za offenkundig das Gegenteil hervorgeht, so ist nichts darauf zu geben, oder Drews ist dieser Meinung nicht.

Zunächst meint er, "Commendetur" am Anfange von I sei allein unverständlich und setze voraus, da $B$ seinem Sebreiber die Redaktion von $H$ rorgelegen haben müsse. Nun ist zwar Commendetur nicht gerade unverständlich, immerhin aber eine ziemliche Härte des Ausdracks, and es 
ist zuzugeben, daB Lather sich wobl anders ausgedruckt haben wurde. Fur die zeitliche tufeinanderfolge der beiden Formulare besagt indes diese Differenz nichts; denn sie kann recht wobl anf der lässigeren Wiedergabe eines gemeinsamen Originals beruhen. Um die sehr naheliegende Frage, ob $\mathrm{H}$ und $\mathrm{I}$ nicht etwa ein solches zugrunde liegt, hat sich Drews uberhaupt nicht gekummert. Er postuliert ganz einfach das Gegenteil. Der zweite Beweis, den er zu bringen versucht, besagt noch weniger. $H$ leitet den zweiten Akt der Ordination mit Facta oratione, I mit Post ein, wie es auch $\mathrm{C}$ and $\mathrm{F}$ tan. Auch daraus soll hervorgehen, daB $\mathrm{H}$ vor I anzusetzen ist. Man sieht hier deutlich, wie Drews nicht vorurteilsfrei untersacht, wie seine ganze Beweisfuhrung eine Verteidigangsrede für die Priorität seines Formalars ist. Aus der konstatierten Tatsache läßt sich uberhaupt nichts folgern. Von ähnlicher Art ist der dritte Beweis. Weil H die Worte cum ordinatoribus ecclesiae nicht enthält, mub es äiter sein als I und alle anderen Formeln. Drews ubersiebt dabei, da $\$$ das miserable, zweideatige Latein. das damit entstanden ist, doch schwerlich einem Originalfornulare Lathers zogeschrieben werden darf. Weiter schreibt in diesem Teile $H$ die Versikel Emitte spiritam tanm ror, während I die Wahl läßt zwischen ihr and einer anderen, die sich in $\mathrm{C}, \mathrm{F}$ and $\mathrm{R}$ findet. Drews sieht anch hierin einen Beweis für die Priorität von H. Bewiesen wird damit freilich nur, da $B$ es den beiden echtlatherischen Formularen noch ferner steht als $\mathrm{I}$. $\mathrm{Da} B$ der Unterschied zwischen $\mathrm{H}$ und $I$ hier auf die Fluchtigkeit des Abschreibers zuruckgeht, dafur spricht abgesehen von dem schlechten Latein der Umstand, daB in $\mathrm{H}$ zwar rom Chore and den Ordinanden, nicht aber vom Ordinator and den ihm administrierenden Geistlichen die Rede ist. Das Wort Ordiuator ist bisber noch gar nicht gefallen. Im ersten Teile wird es nicht erwähnt, es bleibt der Phantasie des Lesers freigestellt, sich den za denken, der die Ordinanden der Furbitte der Gemeinde empfiehlt. Im zweiten Teile erfabren wir noch weniger von ihm und seiner Tätigkeit. $\mathrm{Dab}$ dieser Zustand von $\mathrm{H}$ fur Lathers Verfasserschaft sprechen soll, wird wohl nur wenigen Auserlesenen rerständlich sein. Im Anfange des dritten 
Teiles bringt I fast wörtlich den Text von $C$ und $F$, während es bei $H$ heibt: ordinator ascendat ad gradus superiores. Aach bierin siebt Drews einen Beweis zagunsten seines Formulars; lieber schiebt er Luther einen miserablen Text unter, als daß er anf seine Meinung verzichtet. Ein besonderes Kriteriam der Priorität ist ihm natlirlich die in diesem Teile vorhandene Ansprache, die mit der Timotheusstelle I, 4, $4 \mathrm{f}$. beginnt and an die sich die Schriftrerlesang ron Tim. I, 3, 1-7 und Acta 20, $28 \mathrm{ff}$. anschlieBt. DaB bier die Ordinationsrede vor die verordneten Texte gestellt ist und mit ihnen in keinem besonderen Zusammenhange steht, ubersieht er dabei völlig. Einen weiteren Beweis für $\mathrm{H}$ findet er dann in dem Wegfall der anf Acta 20 in I folgenden zweiten Ordinationsansprache. Da F es dem Ordinator freistellt, bei Zeitmangel die ganze Auspracbe wegzulassen, so folgert er, $F$ wisse, daß sie frther kurzer gewesen sei, und wieder steht ihm $H$ an der Spitze sämtlicber Formulare. Später kommen ihm freilich Bedenken; am Schlnsse seiner Untersuchung nimmt er die Frage noch einmal auf mit dem Resultate, dab sich aus dem Ausfall der zweiten Rede kein stichhaltiger Grund gegen die Priorität von $\mathrm{H}$ vor $\mathrm{I}$ geltend machen lasse. Drews hätte besser getan, die Frage zu erörtern: Hat $\mathrm{H}$ oder seine Vorlage etwa die Erlaubnis ron $F$ befolgt and die auf die Texte folgende Ordinationsrede wegzulassen versucht? Fast scheint es so. Das Gelöbnis der Ordinanden muBte damit fallen, da es im engsten $\mathrm{Za}$ sammenhange mit der Rede steht. Aber der Verfasser des Formulars mag nun wohl das richtige Gefuhl gehabt haben, daß etwas fehlt, daß der eigne Anteil des Ordinators an seiner Amtshaudlung nunmehr gar za gering ist, und daB es doch nicht gat angeht zu ordinieren, ohne einige ermahnende Worte an die Ordinanden zu richten. So entstand dann die Ansprache vor den Texten. Dem Verfasser von I scheint die Geschmacklosigkeit dieser Änderung eingeleuchtet zu haben; er strich daber die Rede und setzte dafur den kleinen Abschnitt nach Acta 20 ein, der wobl andeaten soll, dab hier kunftig die Ordinationsrede des lutherischen Originals za bringen ist.

Drews hat noch eine ganze Reihe von „Beweisen“ filr 
seine Bewertung ron $\mathrm{H}$ angefuhrt. Es lohnt nicht, sie za widerlegen. sie sind ron derselben Art wie die bereits erörterten; kleine Abweichungen des Textes, die mit der Flichtigkeit des Abscbreibers sich hinreichend erklären lassen, and die im besten Falle nur sagen, da $B$ ans irgendwelchem Grunde sich $\mathrm{H}$ hier anders ausdruckt als die ubrigen Formulare, werden dazu verwendet, um seine Priorität als gesichert hinzustellen.

Die Frage, ob $\mathrm{H}$ und $\mathrm{I}$ auf ein gemeinsames Original zuruckgehen, läbt sich leider mit absolnter Sicherheit nicht beantworten. Sehr wabrscheinlich ist es. H durfte eine mit ziemlicher Fluchtigkeit angefertigte Abschrift sein, während I schon Sparen der Überarbeitung, und zwar unter Anlehnung an das lutherische Formalar, zeigt. Wer der Verfasser des Originals von $H$ and $I$ gewesen ist, läBt sich nicht sagen. Ich habe frither an Rörer gedacht, der wiederholt als Stellvertreter Luthers ordinierte und der wohl das Bedurfnis nach etwas mehr Selbständigkeit gehabt haben kann, als die stete Benutzung des latherischen Originals gestattete. Der Fandort von I wtirde dem nicht widersprechen. Es kann indes auch ein anderer gewesen sein. Eine sichere Datierang des Originals von $H$ and $I$ and seiner beiden Ableger ist anmöglich. Drews hat zwar auf eine gewisse Äbnlichkeit des Gedankeninhalts des ersten Teiles von $\mathrm{H}$ mit dem Schlusse der Predigt Lathers vom 20. Oktober 1535 aufmerksam gemacht. Das wurde freilich uar einen Terminus post quem and nichts weiter ergeben. Nun ist es aber wenig wahrscheinlich, dab die beiden Teile von $\mathrm{H}$ zueinander gebören, fur das Formular $\mathrm{H}$ ist damit also nichts gewonnen. Fur die Geschichte der protestantischen Ordinationsformalare ist die ganze Frage anch von geringer Bedeutang. Denn als Luthers Arbeit können weder $\mathrm{H}$ noch $\mathrm{I}$. angesehen werden, als die ältesten genuinlutherischen Formeln sind anch heate noch $\mathrm{F}$ und $\mathrm{C}$ za betrachten. Vielleicht bringt ein glucklicher Zufall uns einmal Kunde von einem älteren Formulare des Reformators, auf Grand dessen wir einen genaueren Einblick in die Anfänge der Ordination erhalten. 